\title{
PRACTICAL EVIDENCE OF WEB-BASED IDEA MANAGEMENT SYSTEMS: CLASSIFICATION AND APPLICATION
}

\author{
Elīna Miķelsone, Tatjana Volkova, Elita Lielā \\ BA School of Business and Finance, Latvia \\ mikelsone.elina@gmail.com
}

\begin{abstract}
Multiple information systems have been developed during the last decade to gain more from collaboration, knowledge management and ideas. One type of such tools is the idea management systems (IMS) - a manageable systematic tool to generate and evaluate ideas. However, there is a lack of research which explores what web-based IMS are, and how they materialize practically. To fill the gap, the paper aims to create classification and application description of webbased IMS by adapting the theoretical and empirical research approaches. To achieve this aim, first, scientific papers, book chapters, and proceedings focused on the idea management and IMS were analyzed using a systematic literature review method and content analysis technique. Based on the analyses, several possible classifications of IMS criteria were found. Second, commercially available web-based IMS evaluation was conducted to verify the criteria and to add data-based classification criteria. Analysis of IMS has helped to characterize parities and disparities of web-based IMS. Results prove that IMS could be classified by their application focus - as 'active' and 'passive'. Dominant type is the active IMS. IMS could also be classified by the sources involved in the idea management - internal, external or mixed IMS. The main structural features of the web-based IMS are idea generation, idea evaluation, and idea retention. Results prove that there are no important differences between theoretical and empirical research results.

Key words: Idea Management Systems; Classification, Web-based; Application, Information Technology.
\end{abstract}

\section{Introduction}

Polaris, Rolls-Royce and Pfizer have reached up to $80 \%$ improvements in time to market, creating new patents and service propositions with real revenue realization in excess of $\$ 50$ million thanks to IMS Spigit (Juma, 2018). The idea management system Crowdcity is trusted by clients, such as Intuit, $\mathrm{P} \& \mathrm{G}$, the NHS, and others to engage employees, partners and customers in order to address the unique challenges they face (Crowdcity, 2018). Many organizations all over the world apply web-based IMS to solve their or their client problems. But even as these systems become more frequently applied in the organization, there is a research gap found in literature - that there is no common view on these systems. In previously conducted research (Mikelsone \& Liela, 2015), the authors have developed a definition of IMS based on more than 70 literature sources. Definition developed during the research is that IMS are systematic, manageable tools, tool kits that help users to generate, evaluate and continue this process (that is, repeated idea generation and evaluation). The authors in that research have concluded that there is a great need to research web-based IMS empirically, enriching theoretical findings.

There is a literature base for this research. First, IMS literature gives wide theoretical insights into the idea management and IMS concept, both from systematic (e.g. Bailey \& Horvitz, 2010; Vandenbosch et al., 2006) and structural (e.g. Bassiti \& Ajhoun, 2013; Bergendahl \& Magnusson, 2014; Divakaran, 2016; Narvaez \& Gardoni, 2015) viewpoints. Literature sources regarding systematic IMS perspective focus on the social capital, creativity, cognition etc., but structural IMS perspective focus on the systems, design and the process (Jensen, 2012).

In their previous research, the authors have found that there are multiple pieces of research with a structural perspective that provide a theoretical base for further IMS concept exploration. Most researches that explore the existing IMS look into one or a few systems, while this research studies 108 web-based IMS based on the systematic approach. As a result, this will be the largest web-based IMS study. Previously, Summa (2004) has researched and compared 24 IMS, however, without a systematic approach. Classifications as a topic of IMS literature have been undervalued because there has been no scientific effort to do that up to now.

Focusing on IMS concept exploration, there are 2 main research gaps found in the literature: (1) there is no common view on IMS concept; (2) there are no IMS classifications based on theoretical or empirical research. It is very important to fill these gaps for the following reasons. First, common understanding will help researchers and IMS applicators to understand the basic concept of IMS and what could be reached with their application. Second, the exploration of different IMS classes could benefit future researches and advise end-users practically by providing main disparities of different IMS. The parity (provided by IMS basic concept exploration) and disparity (provided by the classification development) descriptions will help to build an understanding of IMS full potential and highlight different system classes that could provide different benefits.

There is also a personal motivation of the authors for this research. Although there is a great variety of 
web-based IMS and, as previously shown, many wellknown organizations have used them, the situation that was described in the legendary publication 'Capturing the Complexity in Advanced Technology Use: Adaptive Structuration Theory' by DeSanctis and Poole (1994) is still topical: developers and users of the information management systems see potential of these systems but positive outcomes often do not occur consistently and that is one of the reasons why organizations do not use them commonly. That is the reason why it is vitally important to research web-based IMS and their application that could be one of the steps to explore how to apply these systems to increase organizational effectiveness. This paper is one of the steps on the authors' way of exploring web-based IMS application to increase organizational performance and effectiveness within adaptive structuration theory context. And in this way, it is vitally important to characterize web-based IMS practically, not only theoretically.

To fill the gap, the authors apply theoretical and empirical approach with the main aim of developing web-based IMS parity description by creating web-based basic concept explanation and disparity descriptions by creating classifications of web-based IMS. The paper aims to answer the research questions: (RQ1) what is meant by IMS at a conceptual level - main elements; (RQ2) what are the main IMS application approaches found in the literature - possible classification criteria; (RQ3) what is web-based IMS empirically? The answer to these questions implies an answer to the underlying composite question: what are web-based IMS parities and disparities?

This paper proposes that both terms - the idea management (IM) and IMS - are connected terms, which means that the literature about both terms should be researched. But as IM is a wider and more uncertain term and IMS is more determined subterm of IM, both of these terms should be divided. In this paper, IM is understood as a process, but IMS as a tool kit which provides IM. This paper fulfills an identified need to clarify IMS concept by applying theoretical and empirical approaches. The paper creates academic contribution: (1) it is the widest web-based IMS empirical research based on 108 studies and, based on the theoretical and empirical approaches, IMS concept has been clarified; (2) developed the classification of web-based IMS.

\section{Materials and Methods}

Research development is based on a theoretical research method - a literature review and empirical method of web-based IMS research. First, a literature review data collection was conducted in 3 stages: (1) research of scientific databases to explore literature where terms 'idea management' and 'idea management systems' are mentioned, - every term was researched in 7 databases; (2) selection of literature directly about IM, IMS; (3) exclusion of duplicates. The detailed literature source count in different stages is reflected in Table 1.

All the sources that passed stage 3 were used in content analysis to analyze the main elements of IM and IMS. Systematic literature analysis was conducted in 3 step process. First, to make the research process more effective, before the content analysis a review protocol was developed. The development of the protocol is essential to codify as precisely as possible the way studies have been collected to answer specific research questions, namely: (RQ1) what is meant by IMS at a conceptual level - main elements; (RQ2) what are the main IMS application approaches found in literature - possible classification criteria. Second step - data extraction. Third step - information synthesis with the help of thematic analysis. Content analysis instead of thematic analysis was selected because this technique provides not only qualitative but also quantitative insights, which in this case are important to explore the most frequently applied IMS approaches.

\section{Count of the literature sources in stages}

\begin{tabular}{|c|c|c|c|}
\hline & $\begin{array}{l}\text { Stage } 1-\text { in the article title or/and } \\
\text { keywords mentioned terms: }\end{array}$ & $\begin{array}{l}\text { Stage } 2 \text { - directly about (full text } \\
\text { available): }\end{array}$ & $\begin{array}{l}\text { Stage } 3 \text { - unique } \\
\text { sources: }\end{array}$ \\
\hline Scopus & 39702 & 15 & \multirow{8}{*}{82} \\
\hline ScienceDirect & 364611 & 2 & \\
\hline Google Scholar & 3980000 & 33 & \\
\hline Sage Journals & 152934 & 8 & \\
\hline Ebsco & 5129835 & 9 & \\
\hline Emerald & 107825 & 3 & \\
\hline Web of Science & 269 & 52 & \\
\hline Sum & 4645341 & 122 & \\
\hline
\end{tabular}

Source: created by the authors. 
The web-based IMS selection by stages

\begin{tabular}{|c|c|c|c|}
\hline & $\begin{array}{c}\text { Stage 1: keyword 'idea } \\
\text { management system' }\end{array}$ & $\begin{array}{c}\text { Stage 2: web-based IMS } \\
\text { selection }\end{array}$ & $\begin{array}{c}\text { Stage 3: unique IMS } \\
\text { selection }\end{array}$ \\
\hline Capaterra & 116 & 98 & \multirow{2}{*}{108} \\
\hline Google & 129 & 104 & \\
\hline SUM & 245 & 202 & \\
\hline
\end{tabular}

Source: created by the authors.

Based on the analyses, several possible classification criteria have been found by answering to RQ1 and RQ2. Then commercially available webbased IMS evaluation was conducted to verify criteria found in the literature and improved with data-based classification criteria. This method helped to answer RQ3 - what is web-based IMS empirically? It was divided into 2 steps: (1) summary of web-based IMS systems that are commercially available and its content analysis to explore web-based IMS trends and main characteristics; (2) comparative analysis of theoretical and empirical study results. Analysis of systems helped to characterize structural features of web-based IMS that are important for future research to explore webbased IMS relations with organizational effectiveness in adaptive structuration theory context. Data for commercially available web-based evaluation have been selected from product descriptions available on websites of IMS, but information is processed through content analysis. The first step of data selection was to search in the Capaterra database. This database was chosen because it is one of the most comprehensive corporate IT solution databases that are publicly available. In November 2017, the database was searched by entering the keyword 'idea management system'; 116 systems were selected. Additional research was conducted in Google Search by entering the same keywords and looking through the first 100 search pages. In the second stage, only web-based systems were selected and the installed systems were excluded. In the third step, all unique systems that were later analyzed in the research were selected. The web-based IMS selection helped to find 108 webbased IMS (Table 2).

The data analysis was conducted using the content analysis method, which is essential to reach the aim of identifying the main parities and disparities of IMS and creating the methodology. In this paper, one of the most commonly used content analysis models consisting of 3 steps was used (based on Vaismoradi et al., 2013). 1. Preparation - sources are prepared for analysis (selected web-based IMS descriptions in the web-pages of the products) and an analysis protocol is created, based on the inductive and deductive found elements. 2. Organization - completed protocols, nonlinear coding (inductive, deductive). Codes are grouped into categories, selected for relevance to this study. Category network map was created with a global, organizational, and basic category. Repeated category mapping was conducted for disparities to create possible classification criteria. Table 3 shows the category map that helped to systemize protocols.

3. Results - report development, proposing empirically based IMS description and classification proposition. Niknazar and Borgault (2017) have concluded that unification is necessary to better understand the phenomenon under study and improve the common understanding in the research field. That shows that not only classification based on the disparities should be created but also clarified the object itself. The classification is intended to enhance understanding of phenomena related to organizations,

\section{Category map of web-based IMS analysis}

Table 3

\begin{tabular}{|l|l|l|}
\hline Global Category & \multicolumn{1}{|c|}{ Organizational Category } & \multicolumn{1}{c|}{ Basic Category } \\
\hline $\begin{array}{l}\text { IMS } \\
\text { Characteristics }\end{array}$ & $\begin{array}{l}\text { Basic characteristics } \\
\text { (deductive) }\end{array}$ & $\begin{array}{l}\text { Product; Main functions; Main benefits; Price; Clients; Main fields } \\
\text { of uses }\end{array}$ \\
\cline { 2 - 3 } & $\begin{array}{l}\text { IMS characteristics based } \\
\text { on the literature review } \\
\text { (deductive) }\end{array}$ & $\begin{array}{l}\text { Idea generation; Idea evaluation; Idea maintenance/continuation; } \\
\text { Parallelism; Anonymity; Internal IM/ External IM; Transparency; } \\
\text { Active/Passive IM }\end{array}$ \\
\cline { 2 - 3 } & $\begin{array}{l}\text { Data-driven characteristics } \\
\text { (inductive) }\end{array}$ & $\begin{array}{l}\text { Inductive characteristics from IMS descriptions (e.g. dashboards, } \\
\text { collaboration, status tracking, idea creator tracking, idea ranking, } \\
\text { task formats, discussion, feedback, game mechanics, rewards, } \\
\text { process control, involvement monitoring) }\end{array}$ \\
\hline
\end{tabular}

Source: created by the authors. 
management and different objects (Niknazar \& Bourgault, 2017). There are several definitions of the classification. McKelvey (1978) explained that 'classification is identification and assignment of organization forms to formally recognized classes', but Hjorland and Nissen-Pedersen (2005) that it is 'the sorting of objects based on the criteria selected among the properties of classification objects'. These are not conflicting but complementary explanations because classification is identification and assignment of object different classes based on the classification criteria. A single entity could be classified by different classification criteria can be applied. But the value of classification criteria is determined by its alignment with classifier's purpose and there is no universal classification (Hjorland \& Nicolaisen, 2005). In this paper, as the classification possible elements will be applied elements retrieved from the literature review and empirical web-based IMS research using inductive approach - the main separates of the systems are used as classification elements, divided in one phenomena characterized category (classification criteria).

\section{Results and Discussion}

Based on the previous studies, web-based IMS are systematic, manageable tools, toolkits that help users to generate, evaluate, as well as provide continuation of this process (that is, repeated idea generation and evaluation). The main elements of web-based IMS determined by content analysis are: idea generation (including preparation, capture/gathering of ideas, enhancement), idea evaluation (screening, selection), idea maintenance (it is included in the idea generation and evaluation process) and continuation of IM (that includes concept development, distribution of ideas, support during implementation with repeated IM, rewarding and retention). The main application characteristics are transparency, parallelism, anonymity, but approaches - internal IM/ external IM, active/passive IM. Table 4 shows the description of these characteristics, while these elements were evaluated as existing or not existing.

The systems were analyzed based on elements found in the literature, pre-defined categories that described product overall and in data-driven categories. Pre-defined IMS basic research elements based on the research aim were: the product name, main functions, main benefits, price, clients, main fields of uses. Data-driven or inductive characteristics from IMS descriptions, for example, are: dashboards, collaboration, status tracking, idea creator tracking, idea ranking, task formats, discussion, feedback, game mechanics, rewards, process control, involvement monitoring.

IMS main characteristics based on the literature review

Table 4

\begin{tabular}{|c|c|}
\hline Element & Explanation \\
\hline Idea generation & $\begin{array}{l}\text { Idea generation sessions (hybrid brainstorming) including preparation, capture/gathering of } \\
\text { ideas, enhancement (e.g. Korde \& Paulus, 2016; Wooten \& Ulrich, 2015) }\end{array}$ \\
\hline Idea evaluation & $\begin{array}{l}\text { Ability to filter, compare and evaluate ideas submitted into an IMS (screening; selection) } \\
\text { (e.g. Westerski, 2013; Summa, 2004) }\end{array}$ \\
\hline $\begin{array}{l}\text { Idea retention and } \\
\text { continuity of IM }\end{array}$ & $\begin{array}{l}\text { Ability to store ideas, concept development, distribution of ideas, support during } \\
\text { implementation with repeated IM and rewarding, retention (e.g. Dennis \& Garfield, 2003; } \\
\text { Summa, 2004) }\end{array}$ \\
\hline Parallelism & $\begin{array}{l}\text { The ability for members to exchange information simultaneously (e.g. Dennis \& Garfield, } \\
\text { 2003) }\end{array}$ \\
\hline Anonymity & $\begin{array}{l}\text { Enables members to make contributions without attaching their names, which is not } \\
\text { possible when contributions are made verbally (e.g. Dennis \& Garfield, 2003) }\end{array}$ \\
\hline External IM & $\begin{array}{l}\text { External idea generation and evaluation (main IM sources - experts, partners, customers } \\
\text { and other stakeholders (out of an organization) (e.g. Bothos et al., 2012; Tung et al., 2009; } \\
\text { Westerski } \text { et al., 2011) }\end{array}$ \\
\hline Internal IM & $\begin{array}{l}\text { Internal idea generation and evaluation in an organization (main IM source - employees) } \\
\text { (e.g. Bassiti \& Ajhoun, 2013; Deichmann, 2012) }\end{array}$ \\
\hline Mixed IM & $\begin{array}{l}\text { Idea generation and evaluation involving internal and external sources (e.g. Sandstrom \& } \\
\text { Bjork, 2010; Westerski \& Iglesias, 2012) }\end{array}$ \\
\hline Transparency & Transparent evaluation process (e.g. Summa, 2004) \\
\hline Active IM/IMS & Ability to submit ideas that are focused on special needs (focused) (e.g. Gamlin et al., 2007) \\
\hline Passive IM/IMS & $\begin{array}{l}\text { Ability to submit all ideas that come to mind (unfocused). Not providing all IM process } \\
\text { elements (e.g. Gamlin } \text { et al., 2007) }\end{array}$ \\
\hline
\end{tabular}

Source: created by the authors. 
Parities and disparities of web-based IMS

\begin{tabular}{|l|c|l|}
\hline \multicolumn{1}{|c|}{ Element } & Parity/ disparity & \multicolumn{1}{c|}{ Group } \\
\hline $\begin{array}{l}\text { Idea generation; Idea evaluation; Idea retention; Parallelism; } \\
\text { Anonymity; Transparency; Fields of uses; Benefits; Clients }\end{array}$ & Parities & 1-parities \\
\hline External IM; Internal IM; Mixed IM & Disparities & 2 - disparities related to idea sources \\
\cline { 1 - 1 } Active IM; Passive IM & & 3-disparities related to IMS focus \\
\cline { 1 - 1 } $\begin{array}{l}\text { Functions; Dashboards; Status tracking; Idea creator } \\
\text { tracking; Collaboration; Idea ranking; Task formats; }\end{array}$ & & \\
$\begin{array}{l}\text { Discussion; Feedback; Game mechanics; Rewards; Process } \\
\text { control; Involvement monitoring }\end{array}$ & & \\
\hline
\end{tabular}

Source: created by the authors.

Based on the research results, it could be concluded that there are no conflicting insights of pre-defined web-based IMS as systematic, manageable tools, tool kits that help users to generate, evaluate and continue this process (that is, a repeated idea generation and evaluation). This research also proves that the main characteristic elements or structural features of webbased IMS are: idea generation, idea evaluation, idea retention (that is involved in idea generation and evaluation phases). Additional parities are that these systems provide parallelism, anonymity, transparency and are applicable for different kind of idea generation and evaluation. See the list of all parities and disparities in Table 5.

Parities are the elements that were observed in more than $80 \%$ of the web-based IMS. Main disparities highlight the potential elements for classification criteria development. Main disparities were divided into 3 groups: source related elements, application focus related elements, and process function related elements. Based on these 3 groups of elements, the classification criteria were defined. The created classifications: (1) based on the involved IM source (internal, external or mixed IMS); (2) based on the application focus - as 'active' and 'passive'; (3) based on the provided process functions (limited IMS, full IMS, extra IMS. Table 6 shows the disparities and parities, and the description of the classification classes.

Based on the involved IM, the source (internal, external or mixed IMS) provides the insight in the classes of IMS based on the possible idea generators and evaluators. Internal IMS provides an opportunity to involve in IM employees or some departments, external IMS provides an opportunity to involve external sources, for example, crowds, clients etc. (mostly these systems have public platform), mixed IMS provides an opportunity to involve internal and external IM sources, for example, external IM sources create ideas, but internal ones evaluate them or vice versa. Mixed IMS provides a large scale of opportunities on how to manage IM sources.
Based on the application focus, all systems could be classified as 'active' and 'passive'. This classification shows that there are systems that passively collect all ideas not focused, but active IMS provides functions to collect ideas more focused and mostly also includes idea evaluation possibilities.

Based on the provided process functions all systems could be classified as limited IMS, full IMS, extra IMS. Empirical research explored that not all web-based IMS are fully consistent with IMS definition (based on the process functions), but in reality IMS frequently are classified as systems that provide only idea generation (limited IMS) or systems that provide not only IMS process functions, but also some innovation management functions, such as, idea implementation, project management etc. (extra IMS). But the majority of the systems empirically called IMS has IMS process functions described in the theoretical definition- that is idea generation, evaluation, and continuation of IM (full IMS). These are only some of the classifications that could be created for IMS. The authors of the paper hope that this research will embrace a discussion of these classifications with other researchers to create classifications of IMS because there is a lack of research-based classifications that could explain differences between IMS, and consequently have a practical and academical contribution.

\section{Conclusions}

Research results prove that there are no important differences between theoretical and empirical research results. Additionally, researching IMS potential application, it was concluded that these systems are universally applicable for different use specifics and by different users. Main structural features of webbased IMS are idea generation, idea evaluation, idea retention. This paper provides several ways how webbased IMS could be classified by their application focus - as 'active' and 'passive', however, the dominant type of web-based IMS are 'active' IMS that, according to the literature, are more effective (Gamlin et al., 2007). It would be interesting to do 


\section{Disparities and parities of systems}

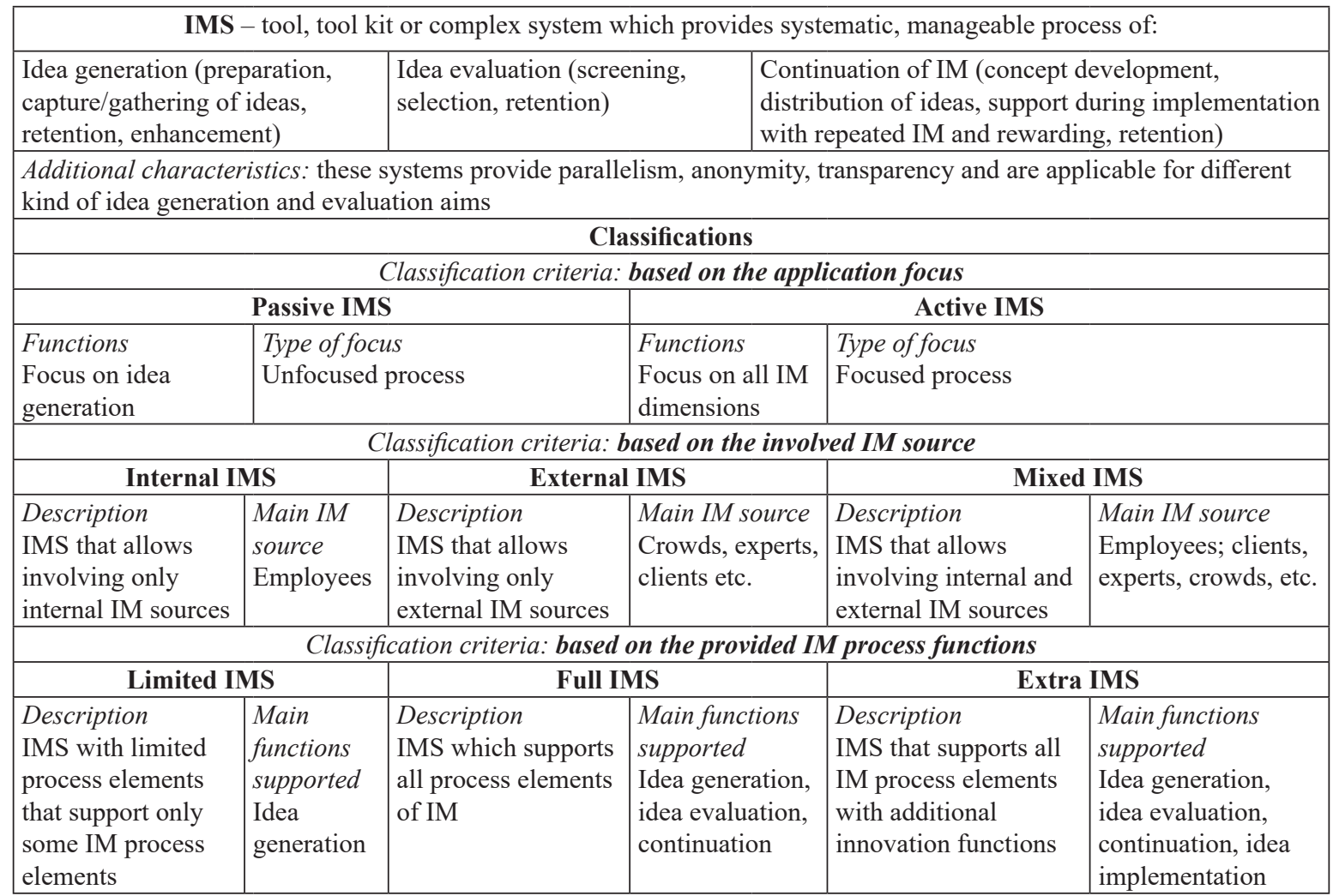

Source: created by the authors.

research on experience of the companies that apply 'passive' IMS and main application benefits, and aims to compare with 'active' IMS. Van den Ende et al. (2015) have observed that in practice there are two main problems in with the idea generation: (1) low quality of ideas; (2) usefulness for organizational strategy. Both problems theoretically could be solved by choosing 'active' IMS with a possibility of creating focused IM processes.

This paper fulfills an identified need to clarify IMS concept applying theoretical and empirical approaches. It creates academical contribution: (1) it is the widest web-based IMS empirical research based on 108 studies; and based on the theoretical and empirical approaches IMS concept has been clarified; (2) developed the classification of webbased IMS. This paper makes an important novel contribution to the literature by characterizing parities and disparities of web-based IMS. It is also the widest research which summarizes the benefits of web-based IMS application. Practical contribution: (1) theoretically and empirically based insight into parities and disparities of web-based IMS that could help to review the potential application of these systems in different other systems; (2) classifications will help to understand some classification criteria that could also be used as criteria for these systems when choosing in practice; (3) classifications highlight benefits/implications of adopting different types of IMS for organizations. These contributions also give managers a richer set of theoretical tools, making them easier to select IMS that are the best for their situation. In the research process and paper development, many future research directions have been identified. First, research could focus on how organizations adapt/customize systems to their own use and implement them in their operations. That would be a great element for case studies that authors are conducting next and is also connected to the adaptive structuration theory. Case studies could provide more comprehensive characteristics of web-based IMS application. This paper concentrated only on commercially available and web-based IMS, but future research could include insights from non-commercial/ private IMS and installed IMS. Additional research should be done on how commercially available systems could be customized and why some organizations create their own IMS. Future research should provide evidence on how different classes of IMS impact organizational results. That also resonates with van den Ende et al. (2015) ideas. The authors hope that this paper will stir up the researchers' interest in classification of IMS, which has been neglected for too long. 


\section{References}

1. Bailey, B.P., \& Horvitz, E. (2010). What's Your Idea? A Case Study of a Grassroots Innovation Pipeline within a Large Software Company. In CHI2010 proceedings on the 28th annual CHI conference on human factors in computing systems, 10-15 April 2010 (pp. 2065-2074). USA, Atlanta, NY: ACM. DOI: $10.1145 / 1753326.1753641$

2. Bassiti, L., \& Ajhoun, R. (2013). Toward an Innovation Management Framework: A Life-cycle Model with an Idea Management focus. International Journal of Innovation, Management and Technology, 4(6), 551-559. DOI: 10.7763/IJIMT.2013.V4.460.

3. Bergendah, M., \& Magnusson, M. (2014). Combining collaboration and competition: a key to improved idea management? European Journal of International Management, 8(5), 528-547. DOI: 10.1504/ EJIM.2014.064603.

4. Bothos, E., Apostoulou, D., \& Mentzas, G. (2012). Collective intelligence with web-based information aggregation markets: The role of market facilitation in idea management. Experts Systems with Applications, 39(1), 1333-1345. DOI: 10.1016/j.eswa.2011.08.014.

5. Crowdcity. (2018). Costumers. Retrieved February 25, 2019, from https://crowdicity.com/customer-stories.

6. Deichmann, D. (2012). Idea Management: Perspectives from Leadership, Learning, and Network Theory. Doctoral dissertation, ERIM.

7. DeSanctis, G., \& Poole, M.S. (1994). Capturing the Complexity in Advanced Technology Use: Adaptive Structuration Theory. Organization Science, 5 (2), 121-147.

8. Divakaran, K.P.P. (2016). When users offer up ideas: how to evaluate them. Journal of Business Strategy, 37(5), 32-38. DOI: 10.1108/JBS-05-2015-0046.

9. Gamlin, J.N., Yourd, R., \& Patric, V. (2007). Unlock creativity with 'active' idea management. Research Technology Management, 50(1), 13-16. DOI: 10.1080/08956308.2007.11657413.

10. Hjorland, B., \& Nicolaisen, J. (2005). The Epistemological Lifeboat: Epistemology and Philosophy of Science for Information Scientists. Copenhagen: Danmarks Biblioteksskole.

11. Hjorland, B., \& Nissen-Pedersen, K. (2005). A substantive theory of classification for information retrieval. Journal of Documentation, 61(5), 582-597. DOI: 10.1108/00220410510625804.

12. Korde, R., \& Paulus, P.B. (2016). Alternating individual and group idea generation: Finding the elusive synergy. Journal of Experimental Social Psychology, 70, 177-190, DOI: 10.1016/j.jesp.2016.11.002.

13. McKealvey, B. (1978). Organisational systematics: taxonomic lessons from biology. Management Science, 24 (13), 1428-1440. DOI: 10.1287/mnsc.24.13.1428.

14. Mikelsone, E., \& Liela, E. (2015). Discussion on the Terms of Idea Management and Idea Management Systems. Journal of Regional Formation and Development Studies, 3(17), 97-110.

15. Naravaez, J.L.M., \& Gardoni, M. (2015). Harnessing idea management in the process of technology transfer at Canadian Space Agency. International Journal on Interactive Design and Manufacturing, 9(33), 1-11. DOI: 10.1109/IDAM.2014.6912694.

16. Niknazar, P., \& Bourgault, M. (2017). Theories for classification vs. classification as theory: Implications of classification and typology for the development of project management theories. International Journal of project Management, 35, 191-203. DOI: 10.1016/j.ijproman.2016.11.002.

17. Jensen, A. (2012). A literature review of idea management. In the $9^{\text {th }}$ NordDesign conference proceedings DS 71 of NordDesign, Aarlborg University, Denmark. 22-24 August, 2012, Retrieved February 25, 2018, from https://www.designsociety.org/publication/35610/a_literature_review_of_idea_management.

18. Sandstrom, C., \& Bjork, J. (2010). Idea management systems for a changing innovation landscape. International Journal of Product Development, 11 (3/4), 310-324. DOI: 10.1504/IJPD.2010.033964.

19. Summa, A. (2004). Software tools to support innovation process-focus on idea management. Working paper (29). Helsinki University of Technology: Innovation Management Institute.

20. Tung, W.F., Yuan, S.T., \& Tsai, J.R. (2009). A custom collaboration service system for idea management of mobile phone design. Human Factors and Ergonomics in Manufacturing, 19(5), 494-509. DOI: 10.1002/ hfm.20147.

21. Vaismoradi, M., Turunen, H., \& Bondas, T. (2013). Content analysis and thematic analysis: Implications for conducting a qualitative descriptive study. Nursing \& Health Sciences, 15(3), 398-405. DOI: 10.1111/ nhs. 12048 .

22. Van den Ende, J., Frederiksen, L., \& Prencipe, A. (2015). The front end of innovation: Organizing search for ideas. Journal of Product Innovation Management, 32(4), 482-487. DOI: 10.1111/jpim.12213.

23. Vandenbosch, B., Saatcioglu, A., \& Fay, S. (2006). Idea management: A systematic view. Journal of Management Studies, 43(2), 259-288. DOI: 10.1111/j.1467-6486.2006.00590.x. 
24. Westerski, A. (2013). Semantic Technologies in Idea Management Systems: A Model for Interoperability, Linking and Filtering. Doctoral dissertation. Spain: Universidad Politécnica de Madrid.

25. Westerski, A., \& Iglesias, A. (2012). Mining sentiments in idea management systems as a tool for rating ideas. In International Conference on the Design of Cooperative Systems (COOP2012) of the Large-Scale Idea Management and Deliberation workshop, 30 May - 1 June, Marseille, France. Retrieved February 25, 2018, from http://www.gi2mo.org/files/papers/coop2012/opinions_coop2012_paper.pdf.

26. Westerski, A., Iglesias, C.A., \& Nagle, T. (2011). The road from community ideas to organisational innovation: a life cycle survey of idea management systems. International Journal of Web Bases Communities, 7(4), 493-506. DOI:10.1504/IJWBC.2011.042993.

27. Wooten, J.O., \& Ulrich, K.T. (2015). Idea Generation and the Role of Feedback: Evidence from Field Experiments with Innovation Tournaments. Production and Operations Management, 26(1), 80-99. DOI: $10.1111 /$ poms.12613. 